\section{Voiding Difficulty and Recur- rent Urinary Tract Infections Secondary to Retained Surg- ical Sponge after Open Simple Prostatectomy}

Sir,

A 58-year male presented to our hospital with complaints of voiding difficulty and recurrent urinary tract infections (UTIS), after undergoing simple open prostatectomy for benign prostatic hyperplasia at some private setup, four months back. Patient had several episodes of fever with chills and severe dysuria. He received antibiotic treatment on prescription of indexsurgeon, but the symptoms were not relieved. On examination, there was mild tenderness in the suprapubic region. Urinalysis revealed frank pyuria. His haemoglobin was $10.5 \mathrm{~g} / \mathrm{dl}$ and total leucocyte count (TLC) was $12,500 / \mathrm{mm}^{3}$. Blood glucose and renal function tests were normal. On ultrasonography, there was a hyperechoic mass at the base of the urinary bladder. ACT scan was done; and reported by radiologist as "Iow density mass with areas of calcification within the bladder, possibly representing a vesical calculus." (Figure 1). The CT scan images showed presence of spongiform pattern and mottled calcification within the bladder. Since the presence of air within aspongecan giveitspongiform appearance and mottled calcification may appear, if the sponge has been retained in the body fora longer period of time. ${ }^{1}$ The authors, therefore, had a high index of suspicion of a retained surgical sponge in the bladder. The patient underwent urethrocystoscopy, which confirmed the presence of a large retained sponge in the bladder, which was removed by open cystotomy (Figure 2). The patient made an uneventful recovery. At two months follow-up, he was voiding well and had no complaints.

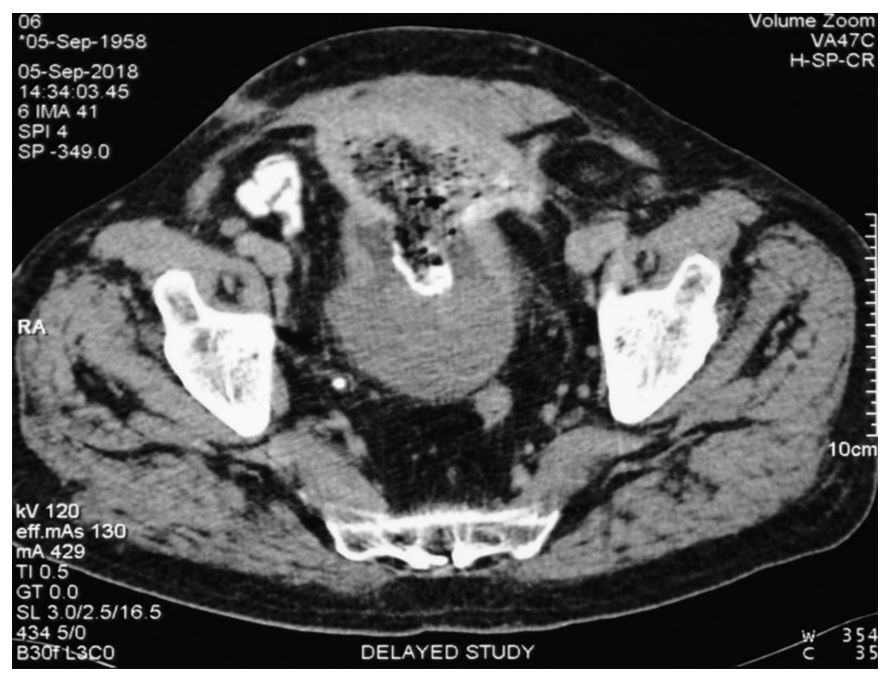

Figure 1: CT scan showing low density vesical mass with spongiform pattern and mottled calcification.

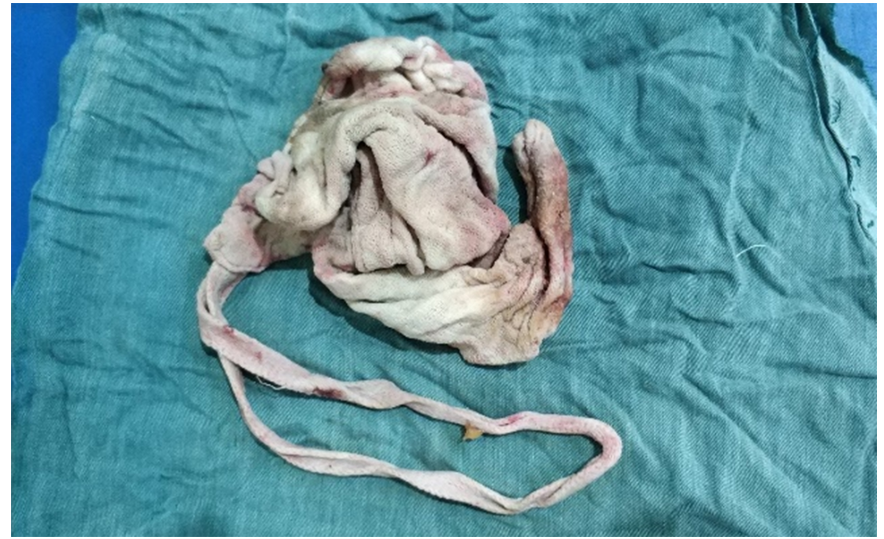

Figure 2: Retained surgical sponge, which was retrieved after surgery.

The retained surgical items (RSIs) occur infrequently. They are associated with significant morbidity, and can have legal implications. RSIs may present as early as the day after surgery or weeks, months, or even years after the original operation. ${ }^{2}$ In urological practice, the surgical guaze pieces are likely to be retained within the bladder after bladder and prostate surgery. ${ }^{3}$ A thorough exploration should be carried out to ensure the removal of all sponges or surgical items before closure of bladder or abdomen. To prevent RSIs, most surgeons rely on the surgical count, which relies heavily on human performance and is prone to error, especially in the emergency surgeries. From root cause analysis, it appeared that RSIs are mostly a consequence of series of errors at the team level rather than from an individualmistake. ${ }^{4}$ A good teamwork, awareness and communication among all operating room personnel, including surgeons, residents, nursing and allied health staff, is necessary to optimise the counting process and prevent RSIs. ${ }^{5}$ In summary, RSIs should be kept in the differential diagnosis of chronic lower urinary tract problems after a major surgery on pelvic organs.

\section{CONFLICT OF INTEREST:}

The authors declared no conflict of interest.

\section{AUTHORS' CONTRIBUTION:}

SR, AR: Data collection, drafting the work and revision for final version to be published.

\section{REFERENCES}

1. Sharma G, Bigelow JC. Retained foreign bodies: A serious threat in the Indian operation room. Ann Med Health Sci Res 2014; 4(1):30-7. doi: 10.4103/2141-9248.126605.

2. Koek AY. Retained surgical sponge presenting four decades later as a rapidly growing soft tissue mass. Case Rep Surg 2020; 2020:1230173. doi: 10.1155/2020/1230173.

3. Rafique M. Vesical gossypiboma. J Coll Phy Surg Pak 2003; 13(5):293-5. doi: 05.2003/JCPSP.293295.

4. Hempel S, Maggard-Gibbons M, Nguyen DK, Dawes AJ, Miake-Lye I, M Beroes J, et al. "Wrong-site surgery, retained surgical items and surgical fires: A systematic review of surgical events". JAMA Surgery 2015; 150(8):796-805. doi: 
10.1001/jamasurg.2015.0301.

5. Feldman DL. Prevention of retained surgical items. Mount Sinai J Med 2011; 78:865-871. doi: 10.1002/msj.20299.

\section{Shoaib Rafique and Ammaar Rafique}

Department of Urology, Care Plus Hospital, Multan, Pakistan
Correspondence to: Dr. Shoaib Rafique, Department of Urology, Care Plus Hospital, Multan, Pakistan

E-mail: shoaib_rafique@hotmail.com

Received: June 10, 2020; Revised: August 07, 2020;

Accepted: August 27, 2020

DOI: https://doi.org/10.29271/jcpsp.2021.09.1137 